\title{
Modelling and Assessing Ad Hoc Networks in Disaster Scenarios
}

\author{
D. G. Reina, S. L. Toral, F. Barrero \\ Electronic Engineering Department \\ University of Seville \\ Seville, Spain \\ d.gutierrez.reina@gmail.com, toral@esi.us.es, \\ fbarrero@esi.us.es
}

\begin{abstract}
Ad hoc networks have been proved to be suitable for disaster scenarios since any infrastructure needs to be deployed in order to establish a wireless network. Routing protocols play an important role in the performance of mobile ad hoc networks. Routing protocols are responsible for deciding how the information is going to move through the network. Although one paramount parameter of ad hoc networks is the mobility of nodes, little effort has been made to evaluate the performance of mobile ad hoc networks under mobility models where the movements of rescue teams during evacuating operations are modelled. The objective of this paper is to evaluate real case disaster scenarios in terms of performance using several well-known routing protocols metrics.
\end{abstract}

Keywords-component; MANETs, mobility model, disaster scenarios, and routing protocols

\section{INTRODUCTION}

Mobile ad hoc networks (MANETs) are wireless networks which are deployed without the need for infrastructure. In MANETs each node acts a router so the nodes are responsible for routing information from source nodes to the destination nodes. However, nodes in MANETs have several restrictions such as limited coverage area, limited lifetime, memory, and cost (Akyildiz et al., 2002). MANETs are suitable whenever the deployment of a new infrastructure is difficult or impossible. Typically applications covered by MANETs are (Sakar et al., 2008): (1) search-and-rescue in disaster situations, (2) defense (army, navy, and air force), (3) health care, (4) academic environment, (5) industrial or corporate environment, (6) home network, and (7) sensor network. In this paper, we focus on the use of MANETs in Disaster scenarios. There have been several proposed approaches in the literature for using MANETs on disaster scenarios (Reina et al., 2010; Reina et al., 2011a). The nodes in MANETs are intrinsically mobile; consequently, some nodes are getting into the coverage area of other nodes, whereas other nodes are getting out of them. As a result, the lifetime of a communication path between two nodes depends on the mobility factors such as the nodes' speed and the direction of movement. Furthermore, a node's power available for transmission determines its coverage area and this parameter affects the temporality of the communication links. Besides, the mobility of nodes causes changes in the network

\author{
N. Bessis, E. Asimakopoulou \\ School of Computing \& Maths \\ University of Derby \\ Derby, UK \\ n.bessis@derby.ac.uk, \\ eleana.asimakopoulou@gmail.com
}

topology. There has been a lot of research on mobility models for MANETs recently (Camp et al., 2002; Bai et al., 2003). The mobility models determine how the nodes move on the target scenarios. In this line, Aschenbruck et al. (2004), proposed a mobility model for disaster scenarios. In this mobility model, the movements of the whole rescue team are modeled. The disaster scenarios are divided into different action areas, and the nodes emulate both mobile and static components of the rescue team, such as ambulances or firefighters. It was included in the mobility generator BonnMotion developed at the University of Bonn, Germany (Aschenbruck et al., 2010a). It provides an output file which can be integrated in Network Simulator 2 (NS-2) (Fall and Varadhan, 2011) in order to run the simulations. Consequently, the performance of ad hoc network under disaster area scenarios can be analyzed.

On other hand, the routing protocol implemented at the nodes play an important role on the performance of MANETs. As a result of the mobility of nodes and their restrictions, multi-hop communications are likely to take place in MANETs. In the case of disaster scenarios the movements of nodes emulate the movements of ambulances taking injured people and other vehicles which take part in rescue operations such as fire engines. In multi-hop communications the nodes should collaborate to each other to establish communication between two nodes that cannot communicate directly by using only one hop. Due to the mobility of nodes, the established communication links are also likely to break frequently. Routing protocols are responsible for reacting whenever a communication link is broken. Depending on the routing protocol, the decision could be to repair the route or to find an alternative or better route to reach the destination. The routing protocols maintain some form of routing tables which contain information related to the neighboring nodes. Attending to the management of these routing tables, routing protocols can be categorized as proactive protocols, reactive protocols, and hybrid protocols (Beraldi and Baldoni, 2003). The proactive protocols maintain the routing table for all the routes, even if they are inactive. However, proactive protocols are not suitable for high density networks and mobile networks due to the fact that maintenance of the routing tables means a high communication flow between nodes causing extra overheads. In contrast, reactive 
protocols maintain only active routes. When a route is inactive or its lifetime is over, the route is removed from the routing table. Hybrid protocols consist of a combination of both earlier mentioned protocols.

The main objective of this paper consists of evaluating the performance of several routing protocols for MANETs under real disaster scenarios, which are a mixture of several types of sub scenarios with different features.

The remainder of this paper is organized as follows. Section 2 describes the disaster area mobility model. Section 3 describes the well-known routing protocol used to evaluate their performances. The description of the real case disaster scenarios is included in section 4 . Simulation results are found in section 5. Finally, conclusions are drawn in section 6.

\section{MOBILITY MODELS FOR RESCUE TEAMS}

\section{A. Mobility Models for Ad Hoc Networks}

Mobility models determine the movements of nodes in the deployed scenario during the simulation time. Several mobility models have been proposed to evaluate the performance of ad hoc networks under different mobility patterns (Camp et al., 2002). So far the Radom Waypoint mobility model is the most used in ad hoc networks. However, it does not represent any real life scenario. Moreover, the nodes do not follow a uniform density since central positions are denser than the others as the simulation time goes on. Random Walk mobility model and Random Direction mobility model solve the mentioned density problem but they still do not represent real situations. Manhattan mobility model allows nodes to move following determined paths like vehicles (Bai et al., 2003). The simulation scenario is divided into different grids and at each intersection nodes have certain probability to turn on. This mobility model is suitable for describing vehicles in urban scenarios. Groups of nodes are taken into consideration in the Reference Point Group mobility model. There is a leader in each group which determines the movements of all nodes in the group. There exists other group mobility models for more specific situations such as Column mobility model, Nomadic mobility model, and Pursue mobility model. Nevertheless, real scenarios are a combination of several mobility patterns. In the same simulation scenario can be found vehicles and people moving at different speeds or different node's density as well. In summary, there is a lack of mobility models for real life scenarios. This lack is partially solved in disaster scenarios since Disaster Area model represents the movement of rescue teams in disaster scenarios (Aschenbruck et al., 2010b).

\section{B. Disaster Area Mobility Model}

The disaster area mobility model is based on a method called separation of the room (Aschenbruck et al., 2010b). Using this method, the disaster scenario is divided into different areas. There areas are: (1) incident site, (2) casualties treatment area, (3) transport zone, and (4) hospital zone.

- Incident site: is the place where the disaster happened. In this place, people normally injured are waiting for being transported to the casualties' treatment areas.

- Casualties' treatment area: consists of two places, (a) patient waiting for treatment and (b) the casualties clearing station. In the first zone, people wait for a first inspection and classification; after that they can be transported to the casualties clearing stations in which patients will be waiting for being transported to a hospital.

- Transport zone: is an area where transport units wait in stand-by areas to transport people to hospitals. The transport units can be either ambulances or rescue helicopters.

- Finally, the technical operational command: is the zone where the rescue operations are commanded and it is usually located in the casualties' treatment area.

Every person participating in the rescue operation belongs to any of the above areas and they are represented by nodes. For example, firefighters take part in the incident site whereas paramedics belong to the casualties' treatment areas in order to first evaluate incoming patients. It is noticeable that each area represents a different scenario where node's density or node's speed are different from one to another. That means the requirements of the routing protocols will be different in each area (Bessis et al, 2011).

Note that, this mobility model does not take into consideration mobility of patients, so it only models the mobility of the rescue teams. Within the rescue team, two types of nodes can be distinguished, static and mobile nodes. The mobile nodes are normally either people carrying patients or vehicles transporting patients to other locations. The maximum speeds of the mentioned types of nodes are clearly different. Mobility of people is significantly slower than mobility of vehicles. The vehicles transport people to hospitals and then go back to the disaster area.

\section{ROUTING PROTOCOLS FOR MANETS}

Since proactive routing protocols are not suitable for MANETs, this section focuses on reactive protocols due to aforementioned mobility of the components of the rescue team.

\section{A. Ad Hoc On-demand Distance Vector}

Ad Hoc On-demand Distance Vector AODV is a reactive routing protocol that uses routing tables with one entry per destination (Perkins and Royer, 1999; Reina et al., 2001b). AODV is based on broadcasting route discovery mechanism. Three types of packets are used in AODV:

- The Request packets (RREQs): RREQs are normally used whenever a source node wants to start 
communicating with a destination node; it floods the network with request packets. A RREQ is forwarded until it reaches the destination node or a node which has a route to communicate with the destination node. For each retransmission the number of hops is incremented. Moreover RREQs are used to form the direct path between the source and the destination nodes.

- The reply packets (RREPs): RREPs are used to respond to the RREQs, and to form the reverse path. To maintain the most recent routing information among nodes, the concept of destination sequence number is used.

- The error packets (RRERs): RRERs are sent whenever a node detects a connectivity failure in the network. It is flooded to all the nodes which form the communication path in order to remove the unreachable routes from their routing tables.

- Hello packets (HELLOs): HELLOs are used to manage local connectivity. The information on the neighbors collected by HELLO packets is stored in a table of neighbors.

AODV is widely the most used reactive routing for ad hoc networks. It is suitable for mobile scenarios.

\section{B. Dynamic Source Routing}

The Dynamic Source Routing protocol (DSR) is a simple and efficient routing protocol specifically designed to be used in multi-hop wireless ad hoc networks of mobile nodes (Jonshon et al., 2001). Using DSR, the network is completely self-organizing and self-configuring, requiring no existing network infrastructure or administration. The DSR protocol allows nodes to dynamically discover a source route across multiple network hops to any destination in the ad hoc network. The DSR routing protocol also uses three of the four mentioned routing packets, RREQ, RREP, and RRER. In contrast, DSR does not use any periodic message like the Hello packets. Each data packet sent then carries in its header the complete, ordered list of nodes through which the packet must pass, allowing packet routing to be trivially loop-free and avoiding the need for up-to-date routing information in the intermediate nodes through which the packet is forwarded. This is one of the main differences between AODV and DSR. While in AODV the routing decision are made node by node, in DSR the source node has all the necessary information to connect with the destination node. It is known as source routing and the node requires a cache memory to implement this routing protocol.

\section{Ad Hoc On- demand Multipath Distance Vector}

Ad Hoc On-demand Multipath Distance Vector AOMDV is a reactive routing protocol which is able to find more than one rout per destination (Mahesh and Das, 2006). It shares several features with AODV. However, in AOMDV the RREQ propagation from the originating node permits to establish multiple reverse paths both at intermediate nodes as well as at the destination. Multiple RREPs traverse these reverse paths back to form multiple communication paths. AOMDV protocol guarantees loopfree, disjoint, and efficient paths using a flood-based route discovery. This routing protocol is suitable for ensuring a minimum path lifetime since alternative route are available whenever a breakage of a route occurs. In contrast, AOMDV causes more overhead due to the extra control packets which participate in finding alternative routes.

\section{MODELING DISASTER SCENARIOS}

In this paper a realistic disaster scenario has been considered (Aschenbruck et al., 2004). The scenario has been already evaluated in terms of mobility model. However, they have not been evaluated under different routing protocols. The following disaster scenario can be modeled using the disaster area mobility model, and the tactical map represented in Fig. 1 (Aschenbruck et al., 2010b).

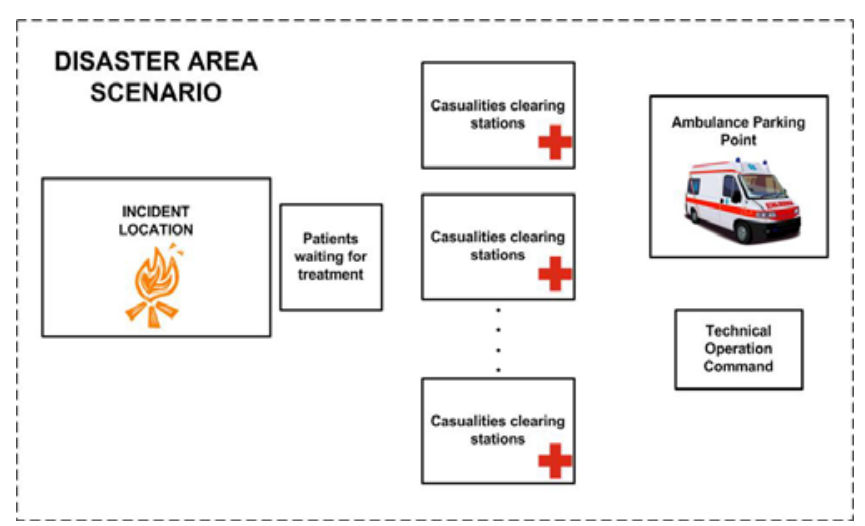

Fig. 1 Modeling Disaster Area Scenarios

The considered scenario is based on a fire in amusement park near Cologne in 2001. One attraction, the rollercoaster, caught fire. In this accident 70 people were injured. The modeled scenario's details can be found in Table 3, (more details about the disaster scenario can be found in Aschenbruck et al., 2004). The number of nodes is $200 \mathrm{~m}$ and total size of the simulation scenario is $550 \times 500 \mathrm{~m}$. Table 1 includes the characteristics of the each area.

Table 1 Simulation areas

\begin{tabular}{|c|c|c|c|}
\hline $\begin{array}{c}\text { Area } \\
\text { (size) }\end{array}$ & $\begin{array}{c}\text { Mobile } \\
\text { Nodes }\end{array}$ & $\begin{array}{c}\text { Static } \\
\text { Nodes }\end{array}$ & Density \\
\hline $\begin{array}{c}\text { Incident site } \\
100 \times 100 \mathrm{~m}\end{array}$ & 60 & 0 & 0.06 \\
\hline $\begin{array}{c}\text { Patient waiting } \\
\text { for treatment } \\
110 \times 70 \mathrm{~m}\end{array}$ & 15 & 15 & 0.042 \\
\hline $\begin{array}{c}\text { Casualties } \\
\text { clearing } \\
\text { station } \\
110 \times 90 \mathrm{~m}\end{array}$ & 0 & 60 & 0.06 \\
\hline $\begin{array}{c}\text { Ambulances } \\
\text { parking } \\
170 \times 90 \mathrm{~m}\end{array}$ & 35 & 5 & 0.0026 \\
\hline Technical & 0 & 10 & 0.025 \\
\hline
\end{tabular}




\begin{tabular}{|c|l|l|l|}
\hline operation & & & \\
$20 \times 20 \mathrm{~m}$ & & & \\
\hline
\end{tabular}

The mobility of nodes is $1-2 \mathrm{~m} / \mathrm{s}$ for people and $5-12 \mathrm{~m} / \mathrm{s}$ for transport vehicles. Considering the density and the mobility of nodes in each area, it is noticeable that each area represents a different simulation scenario by itself. The simulation areas can be classified according to their mobility and density features as in Fig. 2, and it is expected that they perform diffently at each simulation area.

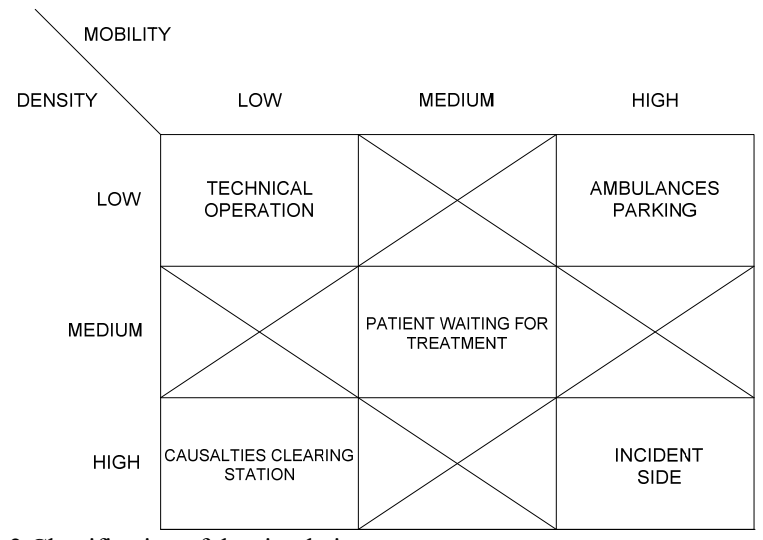

Fig. 2 Classification of the simulation areas

\section{PROPAGATION MODELS}

The propagation models are used to predict the received power signal at the physical layer. Each node has a receiving threshold which determines whether the packet is received or not. Three different propagation models are implemented in ns-2 (Fall and Varadhan, 2011), Free space model, Two-ray ground reflection model, and shadowing model.

\section{A. Free space model}

It assumes an ideal propagation condition. It was proposed by H. T. Friis and the following equation is used to calculate the received signal power

$$
P_{r}(d)=\frac{P_{t} G_{t} G_{r} \lambda}{(4 \pi)^{2} d^{2} L}
$$

Where $P_{t}$ is the transmitted signal power, and $G_{t}$ and $G_{r}$ are the antenna gains of the transmitter and the receiver, respectively. $L$ is the system loss and $\lambda$ is the wavelength. This propagation model is only used in Satellite communications. For this reason, this propagation model is not used in ad hoc networks.

\section{B. Two-ray ground reflection model}

This propagation model considers both the direct path and a ground reflection path. It is shown that this model gives more accurate prediction at a long distance than the free space model.

$$
P_{r}(d)=\frac{P_{t} G_{t} G_{r} h_{t} h_{r}}{d^{4} L}
$$

Where $h_{\mathrm{t}}$ and $h_{r}$ are the transmitter and receiver antennas, respectively. This propagation model shows a faster power decrement as the distance increases. The two-ray propagation model is suitable for urban outdoor scenarios. Most of performance analyses of ad hoc networks have been done using two-ray propagation model.

\section{Shadowing propagation model}

The free and two-ray propagation models predict the received power as deterministic function of the distance. However, the received power at certain distance is a random variable due to multipath propagation effects. The shadowing model consists of two parts. The first one is known as path loss model, which also calculates the mean received power at distance $d$ denoted by $P_{r}\left(d_{0}\right)$.

$$
\left[\overline{\frac{P_{r}(d)}{P_{r}\left(d_{0}\right)}}\right]_{d B}=-10 \beta \log \left(\frac{d}{d_{0}}\right)
$$

Where $\beta$ is called the path loss exponent and is usually estimated by field measurement. The second part of the model is a log-normal random variable. The completed shadowing model is represented by

$$
\left[\overline{\frac{P_{r}(d)}{P_{r}\left(d_{0}\right)}}\right]_{d B}=-10 \beta \log \left(\frac{d}{d_{0}}\right)+X_{d B}
$$

Where $X_{d b}$ is a Gaussian random variable with zero mean and standard deviation. The shadowing model is used in noisy environments and it is also suitable for scenario with a high number of obstacles.

In this paper, the two-ray propagation model and the shadowing model are used to compare the performance of different routing protocols under a disaster scenario. The two-ray propagation model will represent an ideal propagation scenario where nodes can communicate with each other as long as they are within the node's radio transmission range, whereas the shadowing model will represent a nosier scenario with more obstacles and a probabilistic propagation model.

\section{EVALUATION PARAMETERS}

The global simulation parameters can be found in Table 2 .

Table 2 Simulation Parameters

\begin{tabular}{|l|l|}
\hline \multicolumn{1}{|c|}{ Simulation Parameter } & \multicolumn{1}{c|}{ Value } \\
\hline MAC Protocol & 802.11 \\
\hline Propagation model & $\begin{array}{l}\text { Two-ray ground } \\
\text { Shadowing (beta=3.5, sigma }=6.8)\end{array}$ \\
\hline Bit rate & $\mathrm{Mb} / \mathrm{s}$ \\
\hline Carrier frequency & $914 \mathrm{MHz}$ \\
\hline Maximum transmit power, $\mathrm{W}$ & $0.282 \mathrm{~W}$ \\
\hline Interface queue type & $\begin{array}{l}\text { Queue/DropTail/PriQueue (AODV } \\
\text { and AOMDV) } \\
\text { CMUPriQUEUE (DSR) }\end{array}$ \\
\hline $\begin{array}{l}\text { Maximum number of packets in } \\
\text { queue }\end{array}$ & 50 \\
\hline Traffic pattern & Constant Bit Rate (CBR) \\
\hline
\end{tabular}




\begin{tabular}{|l|l|}
\hline Transport protocol & User Datagram Protocol (UDP) \\
\hline $\mathrm{N}^{\circ}$ Connections & $50(10$ for each area) \\
\hline Packet size & 512 Bytes \\
\hline Packet generation rate & $4 \mathrm{pck} / \mathrm{s}$ \\
\hline Propagation model & Two-ray and shadowing \\
\hline Simulation time, $\mathrm{s}$ & $300 \mathrm{~s}$ \\
\hline Mobility Model & Disaster Area Mobility Model \\
\hline Nodes' speeds & $\begin{array}{l}1-2 \mathrm{~m} / \mathrm{s} \text { for people, } 5-12 \mathrm{~m} / \mathrm{s} \text { for } \\
\text { vehicles }\end{array}$ \\
\hline Node's coverage area & $30 \mathrm{~m}$ \\
\hline
\end{tabular}

The following metrics were considered to compare the performance of the routing protocols. These metrics have been chosen since they evaluate the main parameters which impact on the performance of routing protocols. These metrics are widely used to evaluate routing protocols.

- Throughput: total data packets received successfully by their destinations divided by the simulation time.

- Packet Delivery Fraction (PDF): PDF is the ratio between the number of packets originated by the application layer sources and the number of packets received by the destinations. It will describe the loss rate that will be seen by the transport protocol.

- Normalised Routing Load (NRL): The number of routing packets transmitted per data packet delivered at the destination.

- Average end-to-end delay (E2E): This includes all possible delays, caused by buffering during routing discovery latency, queuing at the interface queue, and retransmission delays at the MAC, propagation and transfer times.

- Number of hops: the average number of hops in the routing paths from the source node to the destination node.

- Dropped packets: the total number of dropped packets. The packets can be dropped due to timeout in waiting buffers or broken links.

The mobility generator BonMotion (Aschenbruck et al., 2010a) has been used to generate the mobility patterns. These mobility patterns have been integrated in ns-2.34 (Fall and Varadhan, 2011) in order to compare the performance of the routing protocols.

\section{EVALUATION RESULTS}

We have used Kiviat diagrams to compare the performance of the routing protocols. Kiviat diagrams permit to compare different measure using only one diagram.

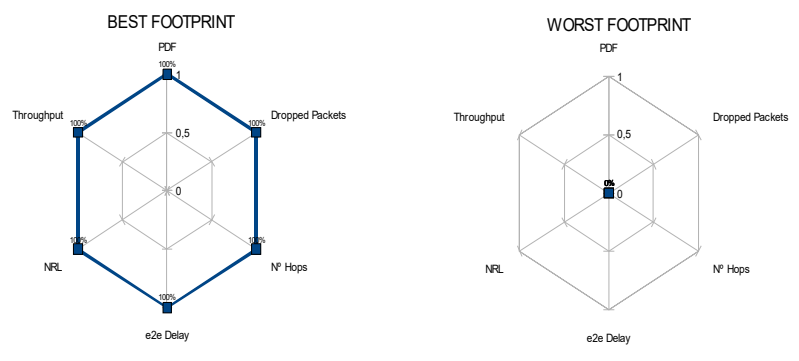

Fig. 3 The best and the worst footprint for a routing protocol

In a Kiviat diagram each axis represents one different measure. However, each axis represents a particular dimension. It is necessary to normalize the axes to ease the interpretation of the results. Each axis is normalized respect to the maximum value obtained in a measure. The Fig. 3 depicts the best and the worst possible footprints for a given routing protocol. Each point in the diagrams has been calculated by averaging out ten different simulations considering random connection among nodes. The simulation results have been divided according to the used propagation model and simulation area.

\section{A. Low number of obstacles (two-ray propagation model)}

In this case the propagation medium is considered to be out of obstacles and noise. Fig. 4 depicts the simulation results for the scenario with higher mobility conditions. The obtained simulation results show that AODV outperform DSR and AOMDV in terms of PDF, throughput and number of dropped packets. However, AOMDV obtained the best results for end to end delay and number of hops. As a result, AODV is the most suitable routing protocol for high mobility conditions as long as the end to end delay fulfills the QoS requirements of the target traffic. DSR exhibits the worst results because of the caused overhead and delay in the communications which in turn cause a decrease in the throughput and an increase of dropped packet due to timeout in the communication buffers. 


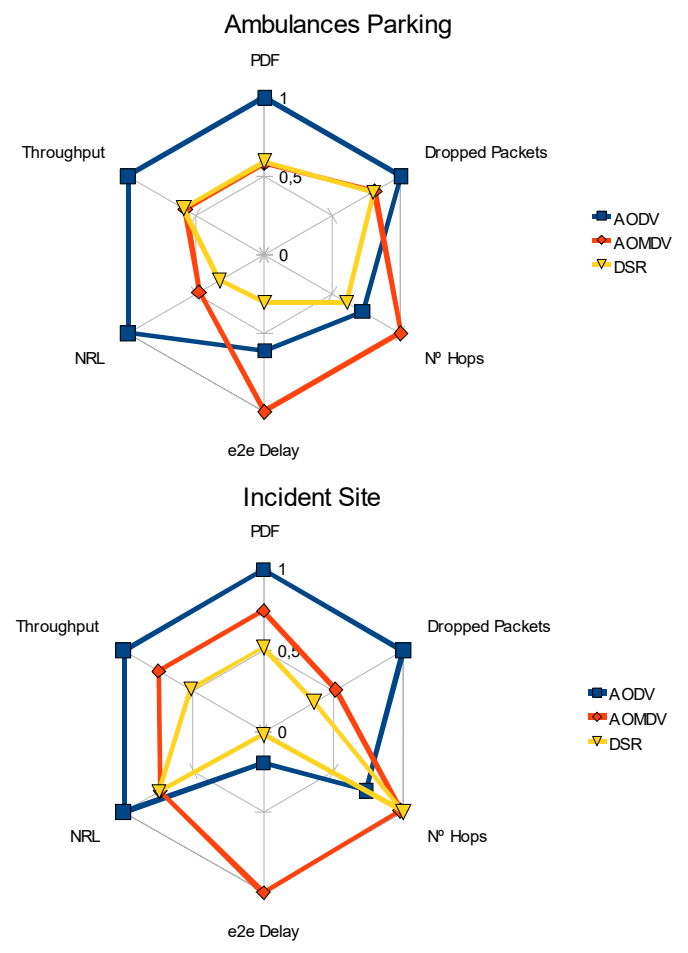

Fig. 4 Simulation results for high mobility areas

As a consequence, for high mobility conditions the most suitable option is to only maintain one route per destination since topological chances are likely to occur very frequently. There is not advantage of considering multiple paths for establishing efficient communications.

Fig. 5 represents the obtained simulation results for the Patient Waiting for Treatment area which has medimum density and medimum mobility conditions. In this case the results of AOMDV are similar to the results obtained by AODV in terms of PDF, dropped packets, and throughput. However, the end to end delay of AOMDV is significantly better. Again DSR suffers from communication delays, but in this case it does not suffer from overheads.

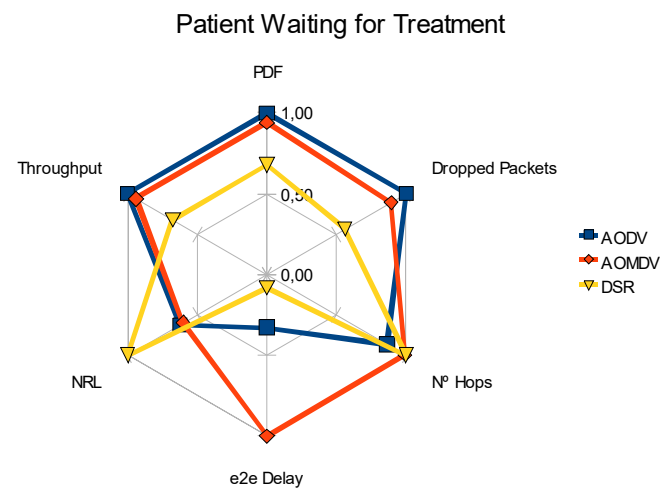

Fig. 5 Simulation results for Patient Waiting for Treatment area
The casualties clearing station area represents a scenario with the highest densiy of nodes and the lowest mobility conditions. The simulation results for this area are shown in Fig. 6. In this case, DSR obtained good results in terms of PDF and throughput maintaining its significant results in term of NRL. Again AOMDV outperforms AODV and DSR in terms of end to end delay.

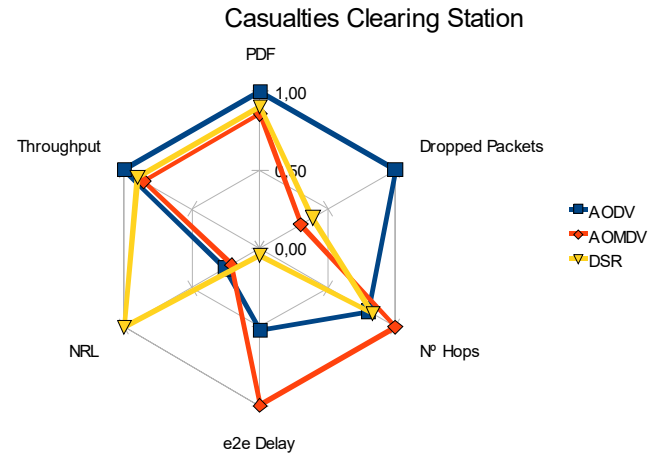

Fig. 6 Simulation results for casualties clearing station area

The technical operation area has not been considered since there are almost no differences in the performance of the routing protocols for this simulation area due to its density and mobility conditions. In the technical simulation area nodes can communicate with each other without any problem obtaining all routing protocol almost the best possible footprint. Finally the Fig. 7 depicts the global simulation results taking into consideration all the simulation areas.

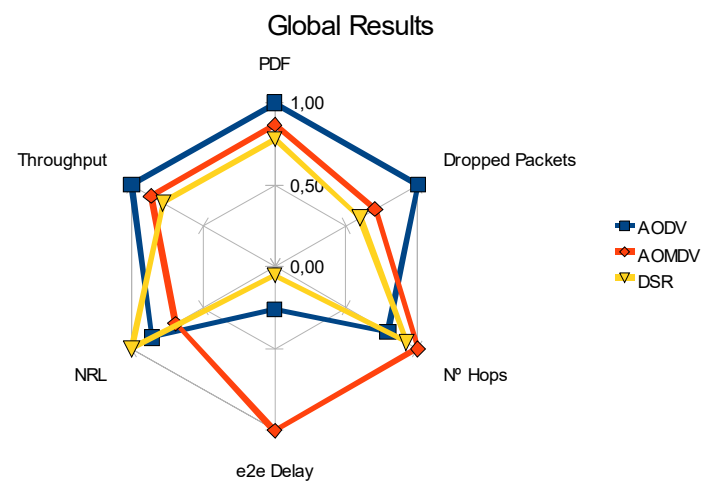

Fig. 7 Global simulation results

With regard to the obtained simulation results AODV outperforms DSR and AOMDV in terms of throughput, PDF, and dropped packets. DSR is the best option in term of overheads. Moreover, AOMDV is the most suitable routing protocol if a low end to end delay is necessary.

\section{B. Noisy Environment (shadowing progation model)}


In this case the shadowing propagation model has been used.

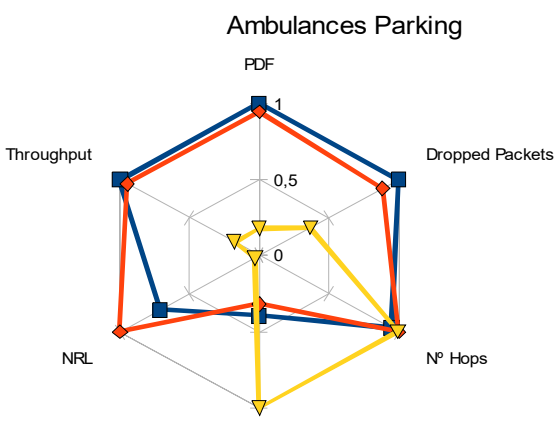

$$
\begin{aligned}
& \Rightarrow \text { AODV } \\
& \Rightarrow \text { AOMDV }
\end{aligned}
$$

e2e Delay

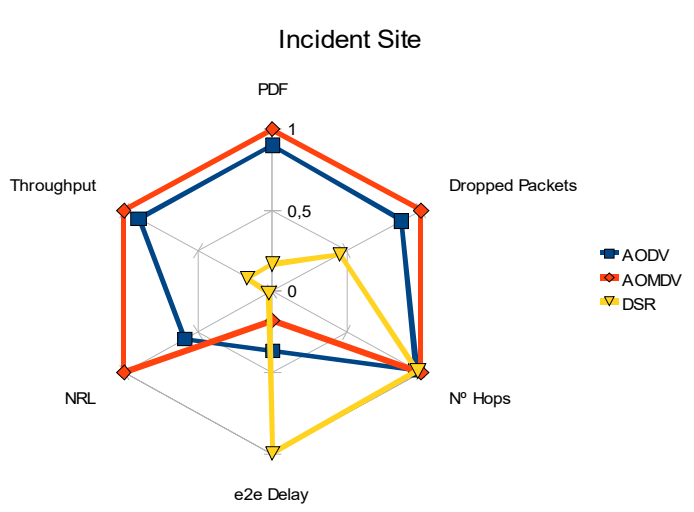

Fig. 8 High mobility areas with a noisy environment

A disaster scenario is likely to be a noisy environment with a high number of obstacles and with difficulties in establishing the communications among nodes. It makes even more difficult multi-hop communications. The Fig. 8 shows the simulation results for the higher mobility areas. The performance of DSR is deficient for these conditions. Although the end to end metric is the highest one, the other metrics show an inefficient performance. The NRL value is prohibitive, which means that in harder conditions DSR makes an aggressive use of routing packets. AOMDV outperforms AODV in terms of NRL, which is an important metric due to the energy consumption which involves the transmission of routing packets.

Regarding the simulation results for the Patient Waiting Treatment area and the Casualties Clearing Station area, AODV outperforms slightly AOMDV in terms of PDF, throughput and dropped packets. However, AOMDV causes less overhead. It is noticeable that with a noisy environment, the performance of AOMDV is delayed as it can be seen in the Fig. 8 to Fig. 11.

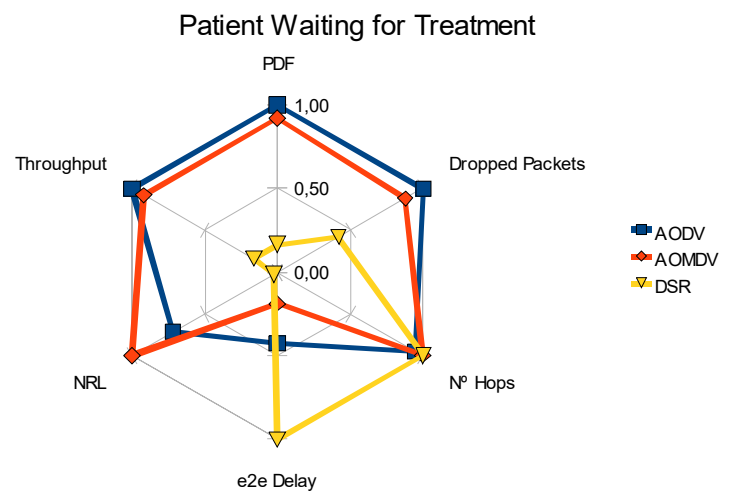

Fig. 9 Patients waiting for treatment area with a noisy environment

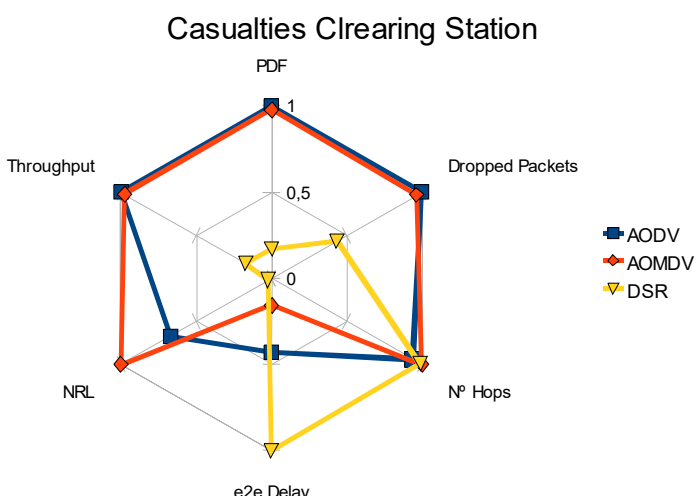

Fig. 10 Casualties clearing station area with a noisy environment

In general, the performance of AODV and AOMDV are similar, the only differences are in terms of NRL and end to end delay. It is clear that DSR is not suitable for noisy environment. This happens whenever nodes have difficulty in establishing communication among them due to lack of connectivity. Again the technical operation area has not been included since the simulation results are very similar for the three considered routing protocols. However, the results for technical operation have been included in the global performance of the routing protocols. 


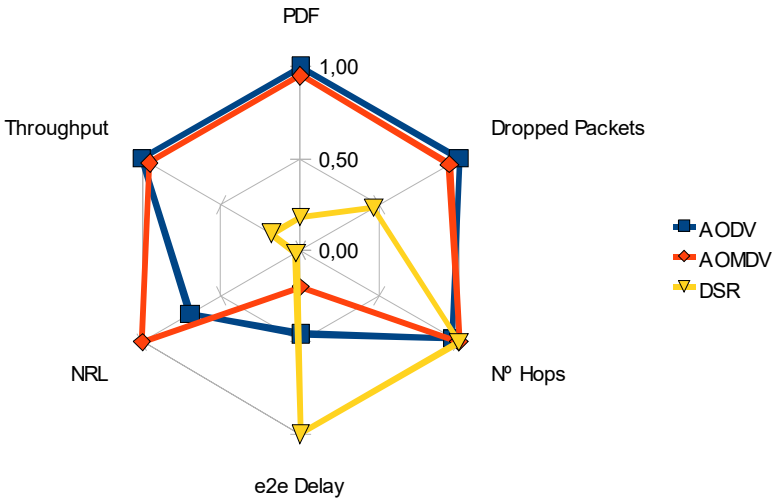

Fig. 11 Global simulation results with a noisy environment

\section{CONLUSION}

In this paper the performance of MANETs routing protocols under a realistic disaster scenario have been evaluated. MANETs represent an attractive alternative to be used in disaster scenarios since they do not need to use any fixed infrastructure. It has been shown that a real scenario is composed of several simulation scenarios which have different requirements. The obtained simulation results change depending on the considered simulation area. Moreover, the radio propagation models also play an important role in the performance of routing protocols. Simulations show that AODV provides the best results in terms of routing metrics. AODMV is good option if end to end QoS is an important requirement as long as the environment is not noisy. The obtained performance of DSR shows that it is not suitable for noisy environments. In summary, there is a necessity for specific routing protocols for rescue teams in disaster scenarios to deal with the difficult conditions and low connectivity exhibited by real disaster scenarios.

\section{REFERENCES}

[1] Akyildiz IF, Su W, Sankarasubramaniam E, and Cayirci E (2002) Wireless Sensor Survey. Computer Networks 5:393-422, 2002.

[2] Sakar SK, Basavaraju TG, and Puttamadappa C (2008) Ad hoc mobile wireless networks: Principles protocols, and applications. Auerbach Publications, Taylor \& Francis Group, pp 24.

[3] Reina DG, Hinojo JM, Toral SL, Barrero F, Cortés F, Soto M, and Marsal E (2010) A wireless in-door system for assisting victims and rescue equipments in a disaster management. Proc. 2010 International Conference on Intelligent Networking and Collaborative Systems INCOS 2010, pp 501-506.

[4] Reina DG, Toral SL, Barrero F, Bessis N, and Asimakopoulou E (2011a) Evaluation of Ad Hoc Networks in Disaster Scenarios. Proc. 2011 International Conference on Intelligent Networking and Collaborative Systems INCOS 2011.

[5] Camp T, Boleng J, and Davies V (2002) A Survey of Mobility Models for Ad Hoc Network Research. Wireless Communications and Mobile Computing 2 (5) 483-502.
[6] Bai F, Sadagopan N, Krishnamachari B, and Helmy A (2003) The IMPORTANT framework for analyzing the Impact of Mobility on Performance Of RouTing protocols for Adhoc Networks. Ad Hoc Networks 1 383-403.

[7] Aschenbruck N, Frank M, Martini P, Tölle J (2004) Human Mobility in MANET Disaster Area Simulation - A realistic Approach". 29 ${ }^{\text {th }}$ Annual IEEE International Conference on Local Computer Network (LCN'04).

[8] Aschenbruck N, Ernst R, Gerhards-Padilla E, Schwamborn M (2010a) BonnMotion- A mobility scenario generation and Anlysis tool. Simutool 2010.

[9] Beraldi R, and Baldoni R (2003) Unicast Routing Techniques for Mobile Ad Hoc Networks. In The handbook of Ad Hoc Wireless Networks. Edited by Mohammad Ilyas. The Electrical Engineering Handbook Series.

[10] Aschenbruck N, Gerhaps-Padilla E, Gerharz M, Frank M, Martini P (2010b) Modelling Mobility in Disaster Area Scenarios. In The 10-th ACM International Symposium on Modeling, Analysis and Simulation of Wireless and Mobile Systems.

[11] Bessis N, Asimakopoulou E, French T, Norrington P, and XHAFA, F. (2010). The Big Picture, from Grids and Clouds to Crowds: A Data Collective Computational Intelligence Case Proposal for Managing Disasters, "1st International Workshop on Emerging Data Technologies for Collective Intelligence (EDTCI-2010)", in conjunction with the 5th IEEE International Conference on P2P, Parallel, Grid, Cloud and Internet Computing (3PGCIC-2010), November 4-6, 2010, Fukuoka, Japan, p.p.: 351-356.

[12] Perkins CE, Royer EM (1999) Ad-hoc on-demand distance vector routing. In Proceeding of IEEE Workshop on Mobile Computing Systems and Applications (WMCSA), pp 1-11.

[13] Reina DG, Toral SL, Johnson P, and Barrero F (2011b) A Reliable Route Selection Scheme based on Caution Zone and Nodes' Arrival Angle, IEEE Communications Letters, 15 (11) 1252-1255.

[14] Jonshon DB, Maltz DA, Broch J (2001) DSR: The dynamic source routing protocol for multi-hop wireless ad hoc networks. In Ad Hoc Networking, ed. by Charles E. Perkins (Addison-Wesley, 2001), pp 139-172.

[15] Mahesh KM, Das SR (2006) Ad Hoc On-demand Multipath Distance Vector Routing. Wireless Communications and Mobile Computing 6 (3) 969-988.

[16] Fall K, and Varadhan K (2011) In The ns Manual (formerly ns Notes and Documentation). Available at http://www.isi.edu/nsnam/ns/nsdocumentation.html [last accessed 07/12/2011]. 
